\title{
Cross-sectional descriptive study of management modalities and quality of life of surgical patients in a palliative care unit
}

\author{
Naing Soe $Y^{\mathrm{a}}$, Amjad $N M^{\mathrm{a}}$, Karim $K \mathrm{~A}^{\mathrm{b}}$ \\ ${ }^{a}$ Department of Surgery, Faculty of Medicine, International Islamic University Malaysia (IIUM), ${ }^{b}$ Department \\ of Surgery, Hospital Tengku Ampuan Afzan (HTAA), Kuantan, Pahang.
}

\begin{abstract}
Introduction: The goal of palliative care is the provision of the best quality of life (QOL) for terminally ill and dying patients. Advances in medical treatment has seen an increase in overall survival of all stages of malignant diseases. This includes advanced and/or inoperable malignancies where management is mainly palliative involving different modalities. Methods: We designed a cross-sectional descriptive study of surgical patients in a palliative care unit in a 1000-bedded teaching hospital in Kuantan, Malaysia. Objectives of this study are: to study the demographic characteristics and indications for admission of surgical patients in palliative care unit, to study the options of treatment modalities and their complications, to identify the barriers in decision making in surgical treatment and finally to objectively assess the quality of life of these patients by utilizing QUALITY OF LIFE (WHOQOL) -BREF -questionnaire. Results: One hundred and one eligible patients $(53 \%)$ male, $(47 \%)$ female of mean age of 54yrs, majority Malay and Chinese patients were included in the study. All patients had malignancies and they were Breast (30\%), Lower gastrointestinal (GI) (24\%), (18\%) upper GI, (15\%) hepato-biliary, and (7\%) pancreatic cancers. Thirty two percents of patients had emergency treatment while the rest had supportive treatment. Barriers to decision making were mainly due to patient factors in $71 \%$, while $12 \%$ was due to the disease presenting at an advanced stage and $15 \%$ due to limitation of care. The final results of overall quality of life rating were shown as poor (1\%), neither poor nor good $(42 \%)$, good $(52 \%)$ and very good $(2 \%)$. Conclusions: Palliative care and end of life decision making from surgical point of view is a delicate issue. Like all other fields in medicine, palliative care must be evidence-based with specific goal directed therapy. Our study shows that we are able to positively impact the quality of life in more than two thirds of our patients. Our aim is to achieve $100 \%$ success. As such, it is imperative to inculcate the goal of palliative care to all grades of health care personnel. 'To cure sometimes, To relieve often, To comfort always' should not be mere words.
\end{abstract}

KEYWORDS: Palliative Care, Neoplasms, Quality of Life, Terminal Care

\section{INTRODUCTION}

The last two decades has seen a paradigm shift among the health care profession in the provision of the needs of the terminally ill and the dying. This has gained world-wide approval in view of the improved survival seen in chronically ill and cancer patients due to advances in medical care. The result of this has been the proliferation of hospice and palliative care programs, education and research activities in most countries.

Corresponding author:

Yan Naing Soe

Department of Surgery

Faculty of Medicine

International Islamic University Malaysia (IIUM)

Jalan Sultan Ahmad Shah,

Bandar Indera Mahkota,

25200 Kuantan, Pahang,

Tel. No. +60168757526

Fax No. (603) 90191959

Email: yynnss@gmail.com
Palliative care service is an evolving field in most developing countries. Malaysia, established it's first Palliative Care Unit in 1995, at the Queen Elizabeth Hospital, Kota Kinabalu, under the Department of Surgery. ${ }^{1}$ Since then, many units have been set up including one at the Hospital Tengku Ampuan Afzan in Kuantan.

Palliative care is recognised as one of the subspecialty with a multi-disciplinary team approach to achieve quality care. Surgical management plays a crucial role in the terminally ill patients and as such a surgeon is a core member of the team.

Like all fields in medicine, palliative care too must be evidence based. Evidence-based practice provides a guide to identify, critically appraise, and use evidence to solve clinical problems. ${ }^{2}$ Practicing surgeons should attain appropriate training in surgery with a focus on care of the terminally ill.

Surgeons may be called to perform either open surgery, minimal access surgery, endoscopy or 
interventional radiology in palliative care patients. This may involve (a) Relief of obstruction, (b) Stopping bleeding, (c) Managing pain, (d) Assisting nutrition support, (e) Providing respiratory support and (f) Controlling infection.

The surgeons are also uniquely skilled to evaluate the risks and benefits of palliative surgery or interventional procedures to achieve quality of living. ${ }^{3}$ Soumitra R Eachempati et al, 2003 highlighted that increasing role of surgeons and surgical intensivists in unique position in modern management of palliative care. ${ }^{4}$

Moreover, future development of training programme will be one of the important domains of higher education and will help to ensure that current and future practitioners of surgery and surgical critical care will maintain the particular knowledge, skills, and attributes necessary for the management of complex surgical patients.

Surgical work group for palliative care (American College of Surgeons, 2003) stated about challenging role of surgeons in palliative care in terms of wide range of knowledge, experienced clinical skills, communication skills, counselling skills, professionalism, practice-based learning and future post graduate training programme with research projects are vital for the dynamic development of palliative care subspecialty and to achieve our goal: quality care to the patients and family members. ${ }^{5}$

The collecting information about subjective domains of life quality might include interviews or questionnaires. To assess health-related quality of life, standardized questionnaires are most often used. In palliative care, especially during the end of life, patients may not be able to complete an instrument that requires self-report.

Under such circumstances, a proxy or health professional will need to rate the patient's experience. In summary, by systemizing the collection of information on patient symptoms and distress, appropriate interventions can be implemented and patient quality of life can be improved. A dynamic approach in clinical practice is needed by combining systematic assessment with individual patient information and communication. ${ }^{6}$

This research was considered useful for providing a framework of quality of life indicators on which the evaluation of the palliative care patients would be based in the future in our unit.

\section{METHODOLOGY}

We designed a cross-sectional descriptive study of surgical patients in the palliative care unit in a teaching hospital in Kuantan, Malaysia. The objectives of this study were:

a. To study the demographic characteristics and indications for admission of surgical patients in palliative care unit

b. To study options of various treatment modalities and their complications

c. To identify the barriers of decision making in surgical treatment

d. To find out the quality of life of the patient in the palliative care unit by inquiry questionnaires.

The patients were from surgical based units who were assigned for palliative care management. We excluded paediatrics and non-surgical patients. Data collection started from $1^{\text {st }}$ Jan 2012 to 31 Aug 2012 till we obtained the statistically significant number of cases.

After initial identification of eligible patients, we collected the demographic data, indications for admission, physical status of the patient and treatment options chosen. Finally, after two weeks of palliative care treatment, we interviewed the patient, in terms of complications of treatment, barriers in decision making and quality life measure questionnaires (by using QUALITY OF LIFE (WHOQOL) -BREF - questionnaires) ${ }^{7}$.

Graph lay-out was done by Microsoft Excel. We used WHOQOL - BREF scoring system to analyze the quality of life questionnaires.

\section{RESULTS}

Data from a total of 101 eligible patients who met our inclusion criteria were collected. Of these $67 \%$ were Malays, 29\% Chinese, 3\% Indian and 1\% others. $54(53 \%)$ patients were females. The mean age of the cohort was 54 years $(28-89 y r s)$.

Thiry percents of these patients had breast carcinoma, 24\% lower GI malignancy, 18\% upper GI malignancy, $15 \%$ hepato-biliary malignancy, $7 \%$ pancreatic malignancy and 6\% other cancers. Figure 1 shows the distribution of the diseases and their respective WHOQOL - BREF scores. 

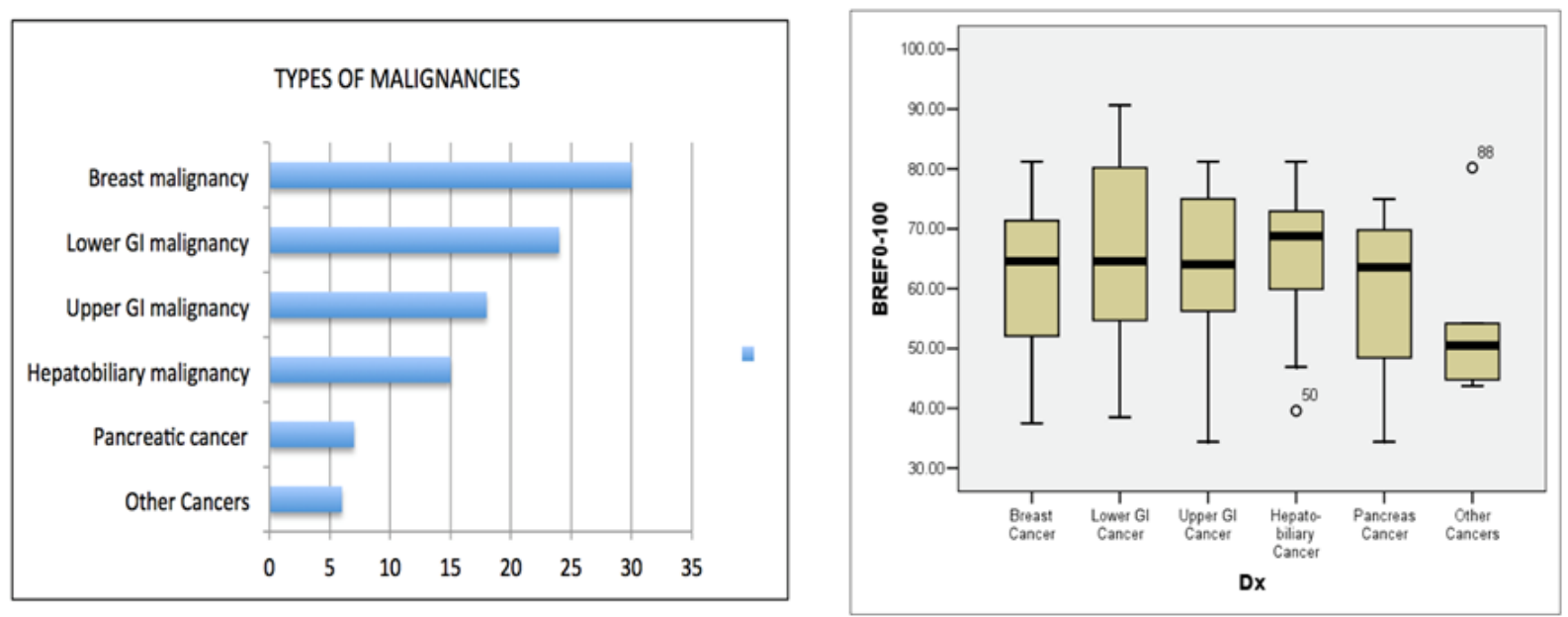

Figure 1 - Types of malignancies and their respective WHOQOL - BREF scores

Indications for admissions were shown in Figure 2 (Chart 1), majority of them were for pain control $(34 \%)$. The other indications were nutrition support (18\%), respiratory support $(16 \%)$, for relief of obstruction(18\%), uncontrolled bleeding as an emergency admission (4\%), end of life decision making and other miscellaneous reasons $(6 \%)$.

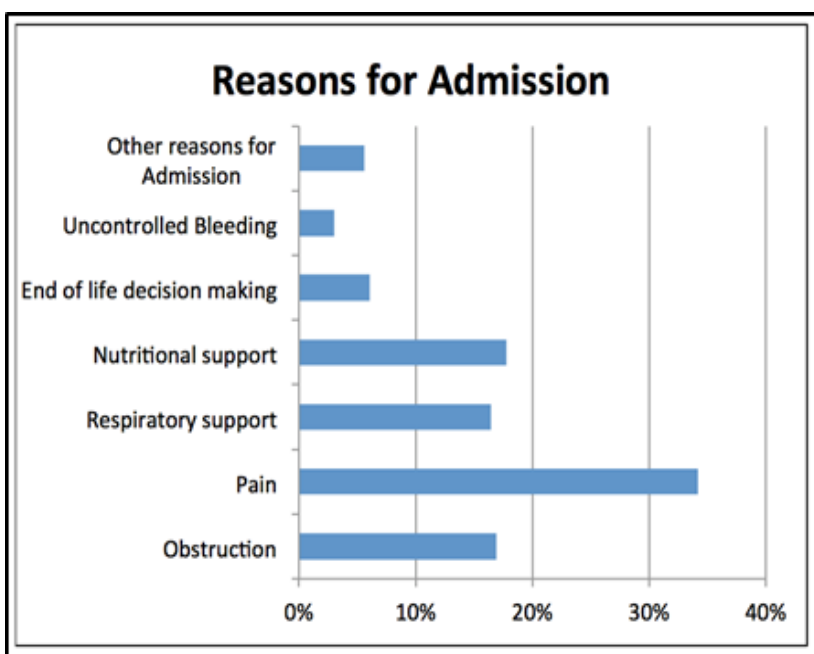

Chart-1

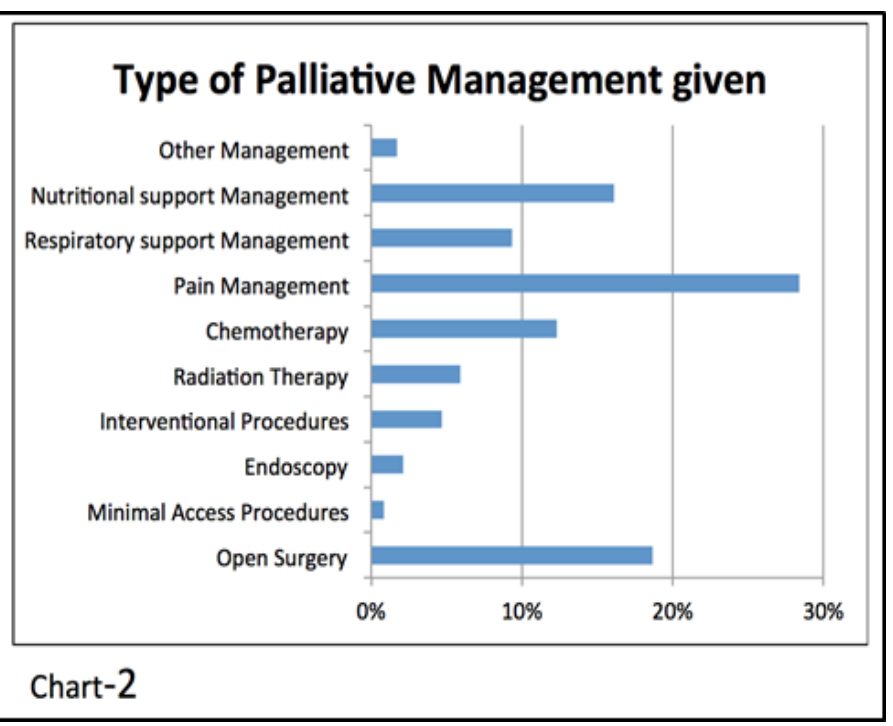

Figure 2 - Indications for admissions and the management modalities

The treatment modalities were shown in Figure 2 (Chart-2). Pain management was $38 \%$, open surgery $18 \%$, nutritional management $16 \%$, palliative chemotherapy $13 \%$, respiratory support management
$9 \%$, palliative radiotherapy $7 \%$ followed by interventional procedures, minimal access surgery, endoscopy and others $5 \%$. 


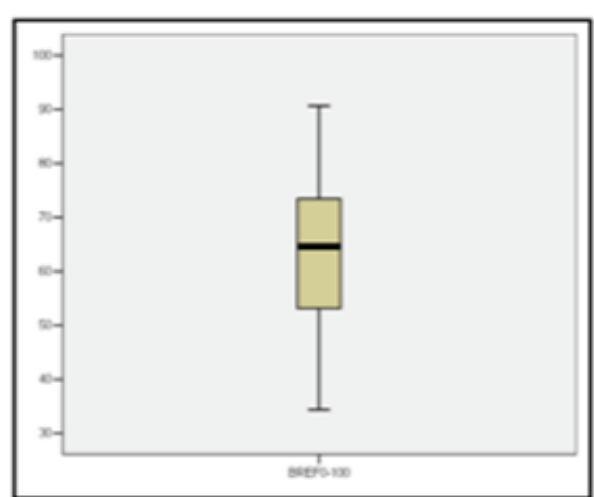

Graph-1: QOL scores in pain management patients (Mean 63.37)

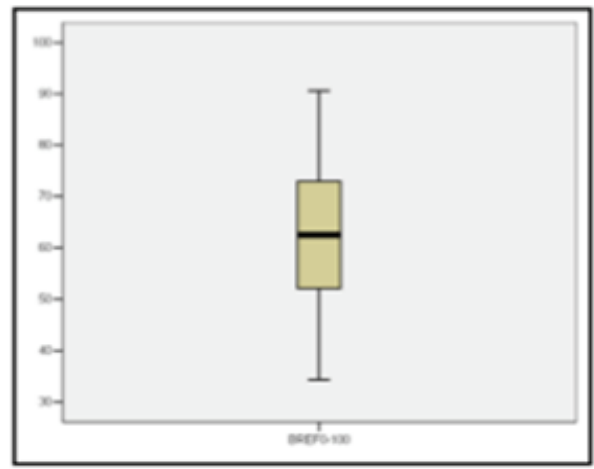

Graph- 3: QOL scores in Nutritional support management patients (Mean 62.44)

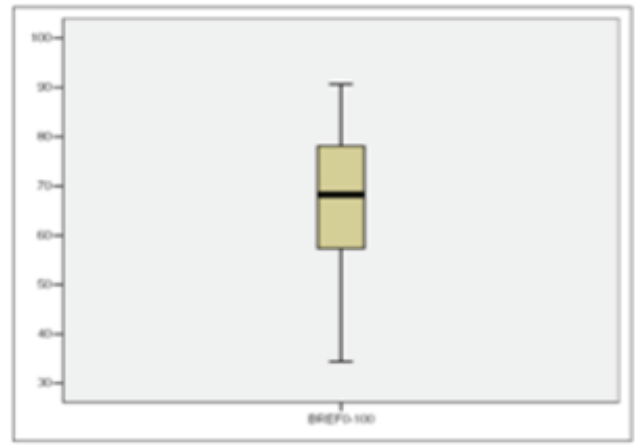

Graph-5: QOL scores in Open Surgery patients [Mean 65.92]

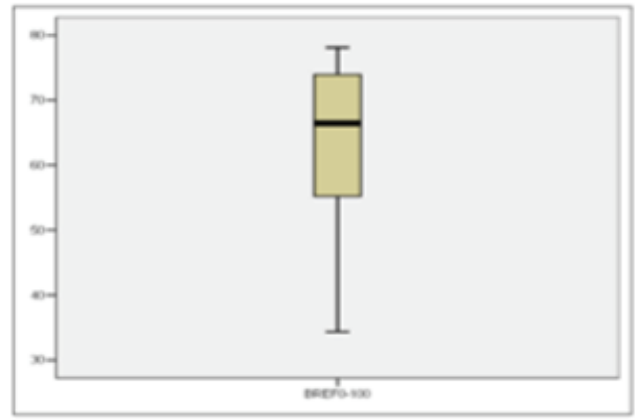

Graph-7: QOL scores in Radiation patients (Mean 63.6)

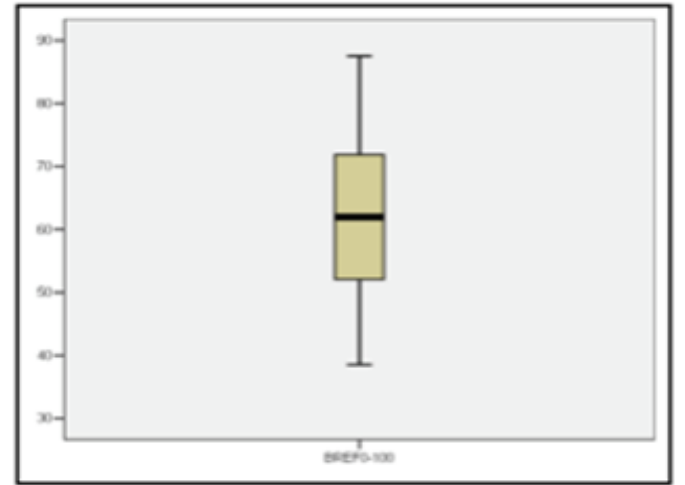

Graph-2: QOL scores in respiratory support management patients (Mean 61.69)

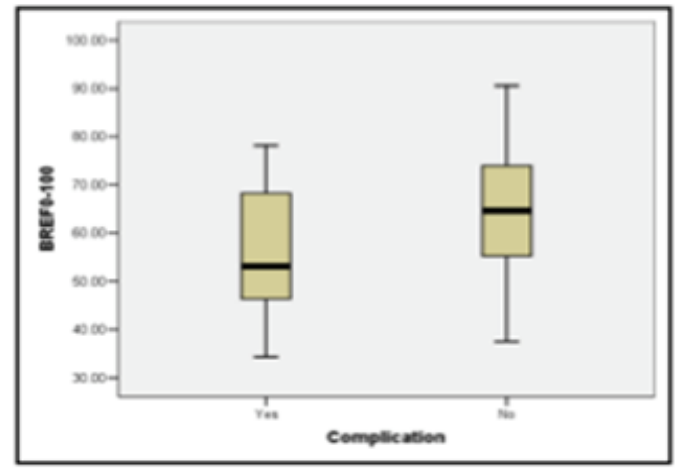

Graph- 4: QOL scores in patients with complications (Mean 52.44) and without (mean- 64)

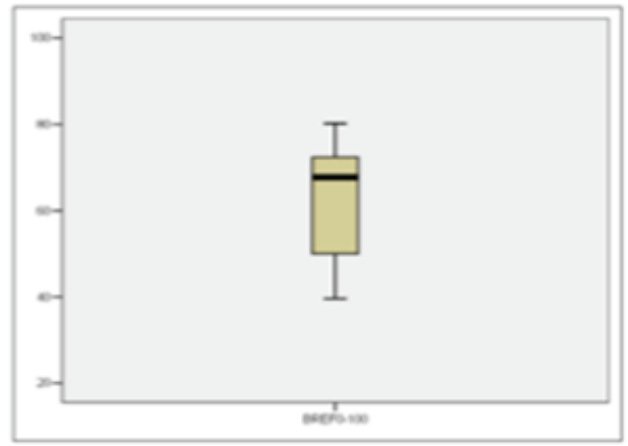

Graph 6: QOL scores in Interventional procedures (Mean 62. 4)

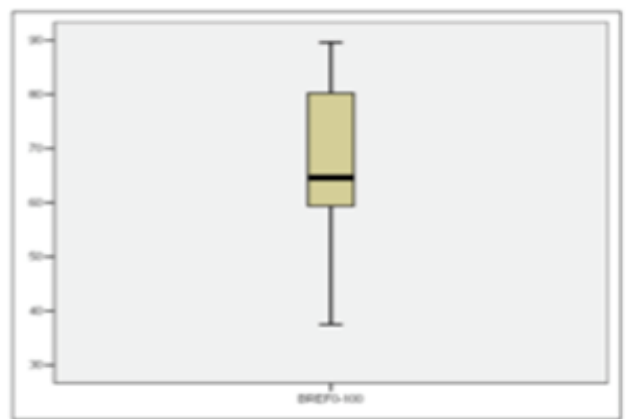

Graph-8: QOL scores in Chemotherapy patients (Mean 66.66)

Figure 3 - WHOQOL - BREF scores for all modalities of treatment and for complications 
Figure 4 shows the barriers that we encountered during decision making for the choice of palliative management. Main source of barrier was patient's reluctance and their cultural \& religious factors comprising the majority. Other significant ones were limitation of facilities and the costs involved. Surgeon's reluctance in making decision was (2-3\%) and others $11 \%$.

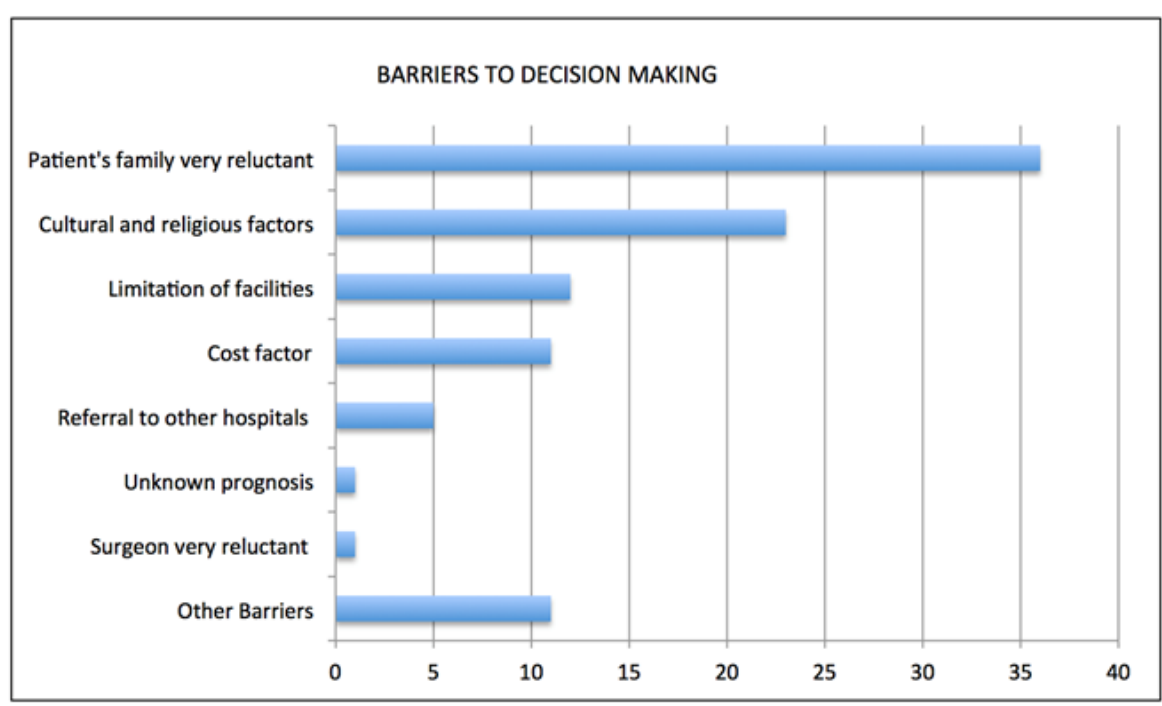

Figure 4 -Barriers for decision-making

Figure 5 represents the functional status of the patients after receiving treatment. Majority of the patient $(53 \%)$ are fully active, $29 \%$ had restricted in movement, $8 \%$ were ambulatory, $9 \%$ needed limited self care and only 1 patient was completely disabled. Each of these groups WHOQOL - BREF scores also highlighted in the above table.

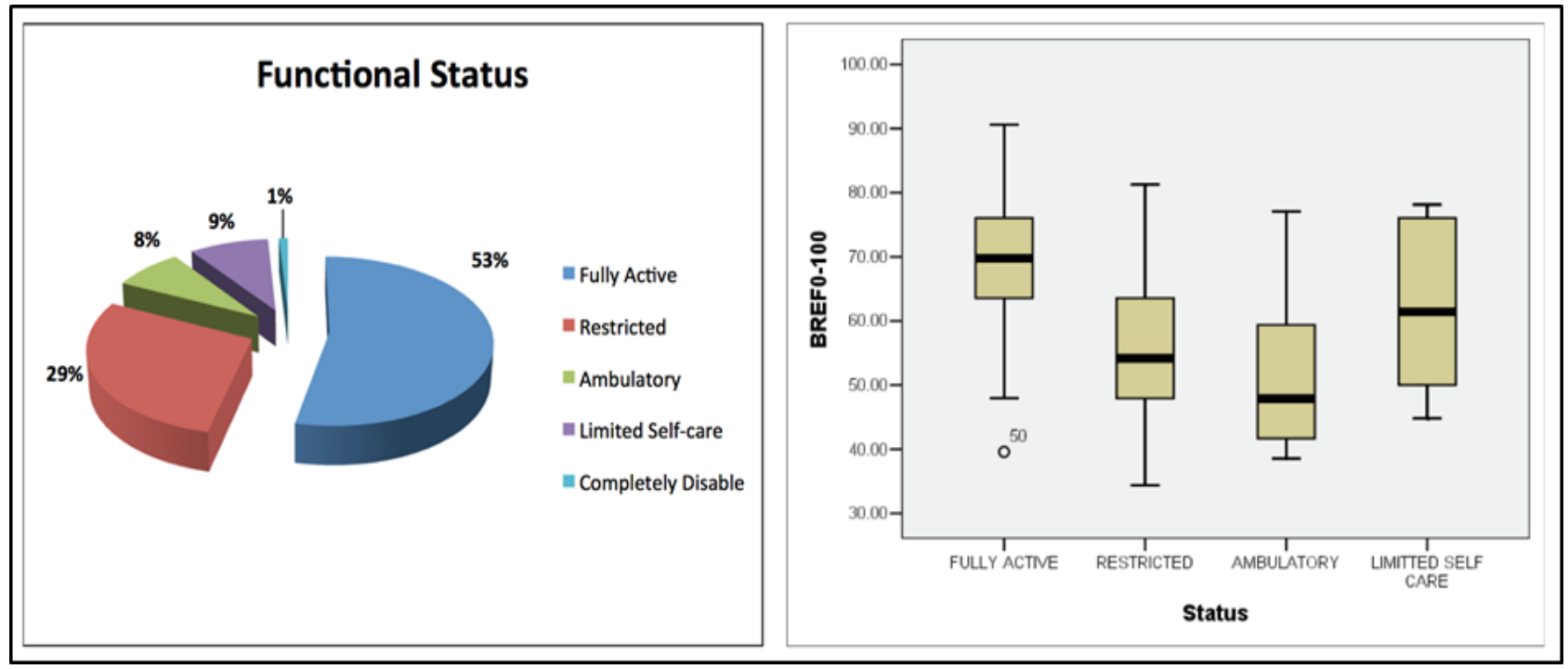

Figure 5 - Functional status of the patients after receiving treatment

Figure 6 shows the patient's self-rating of their QOL according to WHO questionnaires question No- 1., questionnaire No-2 representing the satisfaction of patient's own health and finally the overall QOL rating which is evaluated by questionnaires No-3 to 26. It also highlights the relationship between self rating $(\mathrm{QOL})$ and overall $(\mathrm{QOL})$ rating. 


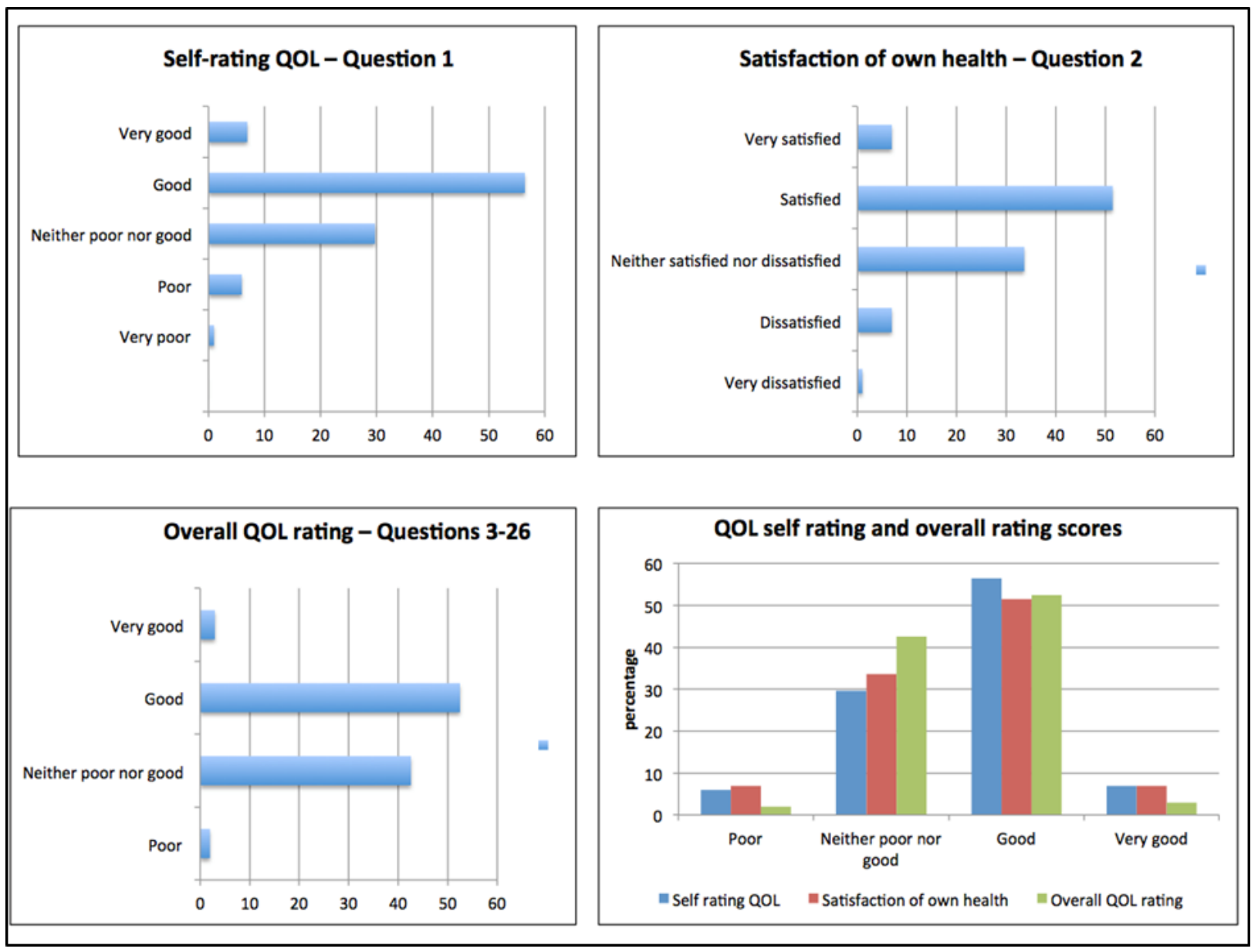

Figure 6- Patient's self-rating of their QOL according to WHO questionnaires

\section{DISCUSSION}

Since two decades ago, the scope of palliative care unit has changed from hospice care to hospital based multidisciplinary team approach. It consists of chronic pain care team, anaesthesiologists, surgeons, interventional radiologists, physiotherapists, nutritionists, psycho-social workers and so on.

In this study, we focused more on surgical patients who had malignancies $(>98 \%)$. It is similar in other countries too. A recent report by the Australian Health Ministry quotes a figure of about $80 \%$ of admissions to palliative-care units being malignancies. ${ }^{8}$ They were warded for various kinds of indications to receive palliative care. From a surgical point of view, the term palliative care is for patients whose disease state cannot be cured and the procedure is carried out to relieve the symptoms thereby facilitating to achieve the optimal quality of life. The ultimate aim being, its benefits must out-weigh the complications. Moreover, the aspects of humanism in surgical practice must be based on empathetic communication and non-abandonment.

With regard to demographic data analysis, 53\% of patients are female and $47 \%$ are male, mean age of
54 years, majority $67 \%$ are Malay followed by $29 \%$ Chinese, $3 \%$ Indian and $1 \%$ others. Breast cancer patients (30\%) and lower gastro-intestinal cancer (24\%) are two major contributor diseases of admission to palliative care unit. It is followed by malignancies of the upper gastro-intestinal tract (18\%), hepato-biliary system (15\%), pancreatic malignancies (7\%) and other soft tissue tumours $(6 \%)$.

Majority of admissions to palliative care unit was for long-term symptomatic supportive treatment (68\%) such as pain control, nutritional support and respiratory support. Significantly, about a third of the admissions (32\%) were emergency admissions requiring interventions like relief of obstructions, control of bleeding, control of sepsis and end of life decision-making where expert opinion and decision sorted.

A recent study revealed that in most emergency admissions, relatively simple medical interventions are necessary where better lines of cooperation between hospitals and the primary care sector may make it possible to perform more of these procedures at home, thereby reducing the need for emergency admissions. ${ }^{9}$ 
In this study, it is shown that emergency surgical intervention was $18 \%$ of which $10 \%$ underwent emergency endoscopy. Others were subjected to minimal access procedures and interventional procedures to treat the immediate life threatening condition of the patients. Twenty eight percents requested admission purely for pain management while $15 \%$ needed nutritional support. A fifth of the patient (20\%) was admitted for palliative chemotherapy and radiotherapy. Majority of patients $(64 \%)$ received palliative care without any complications.

Barriers to palliative care are a relatively common problem. According to a study by Stillman et al., to determine whether the presence of a comprehensive palliative care program in nursing homes would affect the knowledge and attitudes of the staff, their intervention group was able to identify many problems in delivering palliative care than their control group. ${ }^{10}$ We too encountered various types of barriers in our aim to achieve a successful outcome in our care. In this study, we categorized the barriers to decision making into four main groups. They are (a) Patient factors (personal decision, cultural, religious and social factors), (b) Late presentations, (c) Limitation of care (referral to other center) and finally (d) Decision from surgical point of view and prognosis "Full step".

The patient factor contributed most as a major barrier to decision making in palliative care management. Patients themselves or their family members or their care-takers $(35 \%)$ were reluctant to give consent for palliative procedures. This is noted in other studies where patient's denial of having pain as denial of their disease process, not to bother the nurse, or putting up with it as part of the disease. ${ }^{11}$ Other contributory factors in our study influencing decision-making resulting in suboptimal care were cultural and religious beliefs (about 23\%) and costs for other reasons (13\%).

Limitation of care $(13 \%)$ and late presentation of primary disease $(12 \%)$ combined was the second largest factor affecting decision making. Finally, surgeon's decision not to proceed with management $(1-2 \%)$ and unknown prognostic factors $(2-3 \%)$ did impact in a minority of patients. Before assessing the patient's quality of life by using WHOQOL-BREF questionnaires (26 questions), we evaluated the functional status of the patient and divided them into five main categories. More than half of the patients $(53 \%)$ were fully active, $29 \%$ of them were restricted, $8 \%$ ambulatory, $9 \%$ limited self care and only $1 \%$ was completely disabled.

In a prospective study on functional status involving 123 patients conducted at three time points: at admission to specialized palliative care, 14 days post-admission, and at discharge or death revealed patients experiencing significant symptom burden and functional loss from admission to discharge or death. Progressive deterioration became more frequent and severe, while physical and cognitive function decreased at all levels. ${ }^{12}$

In this study we used WHOQOL-BREF questionnaires to access four main categories such as. Overall self rating of QOL (based on question No-1 which is a direct assessment question with regard to quality of life of the current situation) results reveled $1 \%$ to be very poor, $6 \%$ poor, $30 \%$ neither poor nor good, $56 \%$ good and $6 \%$ very good.

The results based on question No-2 (question based on patient's satisfaction of his/her own health status), satisfaction of own health rating showed, (1\%) very dissatisfied, (6\%) dissatisfied, (33\%) Neither satisfied nor dissatisfied, (51\%) satisfied and $6 \%$ ) very satisfied.

From our observation as noted in Table 1 , there is a close positive relationship between the patient's (QOL) scoring and the individual type of treatments adminstered. It is indicative that palliative surgery and interventional procedures do have a major positive impact in more than two thirds of patient's quality of life.

Table 1 - Relationship between the patient's (QOL) scoring and the individual type of treatments adminstered

\begin{tabular}{lll}
\hline \multicolumn{2}{l}{ Types of palliative treatment given } & Paatient's QOL Scores (Mean) \\
\hline 1. & Open surgery & 65.92 \\
2. & Interventional procedures & 62.40 \\
3. & Palliative radiotherapy & 63.06 \\
4. & Palliative chemotherapy & 66.66 \\
5. & Pain management & 63.37 \\
6. & Respiratory support & 61.69 \\
7 & Nutritional support & 62.44 \\
\hline
\end{tabular}


Finally the overall QOL rating was concluded by asking 23 questions (Question No. 3 - 36). These are focused on four domains as shown in Table 2.

Table 2: Domains of quality of life questionare

\begin{tabular}{|c|c|}
\hline Domains & Facets incorporated with domains \\
\hline Physical Health & $\begin{array}{l}\text { Activities of daily living } \\
\text { Dependence on medicinal substances and medical aids } \\
\text { Energy and fatigue } \\
\text { Mobility } \\
\text { Pain and discomfort } \\
\text { Sleep and rest } \\
\text { Work Capacity }\end{array}$ \\
\hline Psychological & $\begin{array}{l}\text { Bodily image and appearance } \\
\text { Negative feelings } \\
\text { Positive feelings } \\
\text { Self-esteem } \\
\text { Spirituality / Religion / Personal beliefs } \\
\text { Thinking, learning, memory and concentration }\end{array}$ \\
\hline Social relationship & $\begin{array}{l}\text { Personal relationships } \\
\text { Social support } \\
\text { Sexual activity }\end{array}$ \\
\hline Environmental & $\begin{array}{l}\text { Financial resources } \\
\text { Freedom, physical safety and security } \\
\text { Health and social care: accessibility and quality } \\
\text { Home environment } \\
\text { Opportunities for acquiring new information and skills } \\
\text { Participation in and opportunities for recreation / leisure activities } \\
\text { Physical environment (pollution / noise / traffic / climate) } \\
\text { Transport }\end{array}$ \\
\hline
\end{tabular}

\section{CONCLUSION}

It is impossible to palliate all symptoms of terminally ill patients but based on the results of this study, we are able to alleviate to a significant extent the long term symptoms like pain, nutritional insufficiency and inadequate tissue oxygenation but also emergency life threatening conditions like obstruction, torrential bleeding and life threatening infections.

Palliative care and end of life decision-making from a surgical point of view is a delicate issue. Staging terminally ill patients by initial assessment of patient's physical health, psychological status, review of primary illness and prior treatments, social, environmental and cultural assessment is crucial. It is mandatory to communicate with patient and caretaker prior to decision-making. We must anticipate barriers in decision making, most of which are related to level of health education of patients and caretakers.

As shown in this study, a basic palliative unit can be effective in alleviating the suffering and improving their quality of life in almost two thirds of our patient. With a well structured multidisciplinary team, a vast improvement can be achieved.

\section{REFERENCES}

1. Leong RLB. Palliative Care in Malaysia A Decade of Progress and Going Strong. J Pain Palliat Care Pharmacother 2004; 17:77-85.

2. Stanley KJ, Sawrun D \& Treantafilos M. Ethical Issues and Clinical Expertise at the End of Life. Nurs Clin North Am 2008; 43:259-275.

3. Krouse RS, Easson AM \& Angelos P, Ethical Considerations and Barriers to Research in Surgical Palliative Care. J Am Coll Surg 2003; 196: 3:469-474.

4. Eachempati SR, Miller FG \& Fins JJ. The Surgical Intensivist as Mediator of End-of-Life Issues in the Care of Critically Ill Patients. J Am Coll Surg 2003; 197:847-853.

5. Statement on Principles Guiding Care at the End of Life. American College of Surgeons Committee on Ethical Board February 1998 
meeting. Bulletin of the American College of Surgeons 1998; 83(4):11.

6. Wright M, Wood J, Lynch T \& Clark D. Mapping Levels of Palliative Care Development: A Global View. J Pain Symptom Management 2008; 35:469-485.

7. WHO Quality of Life-BREF (WHOQOL-BREF). Available at http://www.who.int/ substance_abuse/research_tools/whoqolbref / en/

8. Trends in palliative care in Australian hospitals. Available at http://www.aihw.gov.au/ publication-detail/? id=10737420167\&libID=10737420166 ISBN 978-1 74249-210-0; Cat. No. HWI 112; 100pp

9. Hjernstad MJ, Kolflaath J, Lokken AO, et al. Are emergency admissions in palliative cancer care always necessary? Results from a descriptive study. BMJ Open 2013; 3:e002515.

10. Stillman D, Strump N, Capezuti, Tuch H. Staff perceptions concerning barriers and facilitators to end-of-life care in the nursing home. Geriatr Nurs 2005; 26:259-64.

11. Egan M \& Cornally N. Identifying barriers to pain management in long-term care. Nursing Older People 2013; 25:25-31. 